\title{
Towards a nonperturbative path integral in gauge theories
}

\author{
SERGEI V. SHABANOV 円 and JOHN R. KLAUDER \& \\ Department of Mathematics, University of Florida, Gainesville, FL 32611, USA
}

\begin{abstract}
We propose a modification of the Faddeev-Popov procedure to construct a path integral representation for the transition amplitude and the partition function for gauge theories whose orbit space has a non-Euclidean geometry. Our approach is based on the Kato-Trotter product formula modified appropriately to incorporate the gauge invariance condition, and thereby equivalence to the Dirac operator formalism is guaranteed by construction. The modified path integral provides a solution to the Gribov obstruction as well as to the operator ordering problem when the orbit space has curvature. A few explicit examples are given to illustrate new features of the formalism developed. The method is applied to the Kogut-Susskind lattice gauge theory to develop a nonperturbative functional integral for a quantum Yang-Mills theory. Feynman's conjecture about a relation between the mass gap and the orbit space geometry in gluodynamics is discussed in the framework of the modified path integral.
\end{abstract}

1. Motivations. In what follows we consider only gauge theories of a special (YangMills) type where gauge transformations are linear transformations in the total configuration space. Let $\mathcal{X}$ be a configuration space which is assumed to be a Euclidean space $\mathbb{R}^{N}$ unless specified otherwise. It is a representation space of a gauge group $\mathcal{G}$ so the action of $\mathcal{G}$ on $\mathcal{X}$ is given by a linear transformation $x \rightarrow \Omega(\omega) x$. A formal sum over paths for the action invariant under gauge transformations would diverge. To regularize it, Faddeev and Popov have proposed to insert the identity [1]

$$
1=\Delta_{F P}(x) \int d \mu(\Omega) \delta(\chi(\Omega x))
$$

where $\chi(x)=0$ is a gauge condition and $\Delta_{F P}$ is the corresponding Faddeev-Popov determinant, into the divergent path integral measure so that after a change of variables $x \rightarrow \Omega x$ the (divergent) volume of the gauge group can be factorized out. The determinant $\Delta_{F P}$ is calculated by doing the group averaging integral (11).

This formalism has provided a solution to two important problems in the perturbative path integral quantization of gauge theories: The unitarity of the perturbative S-matrix and the construction of a local effective gauge fixed action. Later on it has been observed that the procedure becomes ill-defined beyond perturbation theory [2]. A geometrical reason for the Gribov obstruction is that there is no gauge condition which would provide a global parameterization of the gauge orbit space $\mathcal{M}=\mathcal{X} / \mathcal{G}$ by a set of affine coordinates

\footnotetext{
${ }^{1}$ On leave from: Laboratory of Theoretical Physics, JINR, Dubna, Russia

${ }^{2}$ Also Department of Physics
} 
without singularities [3]. This situation is often rendered concrete in the vanishing or even sign changing of the Faddeev-Popov determinant. An additional problem expected in the nonperturbative regime is the operator ordering ambiguity [4]. When the kinetic energy $\dot{x}^{2}$ is projected on the gauge orbit space $\mathcal{M}$, one gets $\left(\dot{h}, g_{p h} \dot{h}\right)$, where $h$ are local coordinates on $\mathcal{M}$ and $g_{p h}$ is an induced metric on $\mathcal{M}$. The metric is not flat [5]. In order to establish a correspondence between the Dirac operator formalism and the gauge-fixed path integral, the latter must be appropriately defined to take into account the operator ordering in the physical kinetic energy operator. It is known that the formal path integral for motion on a curved manifold is ambiguous [6]. Up to the second order of perturbative Yang-Mills theory, operator ordering corrections have no effect on the physical S-matrix [4]. Their role in a nonperturbative description is unknown. A standard argument used in dimensional regularization, $i \hbar \delta^{3}(0)=0$, (a commutator of canonically conjugated field variables at the same spacetime point, which typically emerges in the operator ordering terms) does not seem to be applicable because the validity of dimensional regularization beyond perturbation theory is not justified [7]. Finally, the space $\mathcal{M}$ may have a nontrivial topology [3]. Due to the locality of the Faddeev-Popov construction, global properties of $\mathcal{M}$ are lost in the path integral. On the other hand the topology of the orbit space may be important for the spectrum of physical excitations. Feynman conjectured that the mass gap in gluodynamics in $(2+1)$ spacetime might be caused by a compactification of certain directions in the configuration space upon an identification of gauge equivalent configurations [8].

In this letter we propose a modification of the procedure (1) which gives a solution to the above problems and leads to a well-defined path integral on the orbit space $\mathcal{M}$. Its validity is demonstrated with a few examples.

2. A modified Kato-Trotter product formula. Let $\hat{H}$ and $\hat{\sigma}_{a}$ be self-adjoint Hamiltonian and operators of constraints, respectively. We also assume $\left[\hat{H}, \hat{\sigma}_{a}\right]=0$ which holds for a sufficiently large class of physically interesting gauge models. Let the gauge group be compact. Consider a projection operator

$$
\hat{\mathcal{P}}=\int d \mu(\omega) e^{i \omega^{a} \hat{\sigma}_{a}}, \quad \int d \mu(\omega)=1, \quad \hat{\mathcal{P}}=\hat{\mathcal{P}}^{\dagger}=\hat{\mathcal{P}}^{2}
$$

The operator (2) projects the total Hilbert space onto the Dirac subspace of gauge invariant states. If the gauge group generated by $\hat{\sigma}_{a}$ is not compact, we take a sequence $c_{\delta} \hat{\mathcal{P}}_{\delta}$ of rescaled projection operators $\hat{\mathcal{P}}_{\delta}$, where $\hat{\mathcal{P}}_{\delta}$ projects on the subspace $\sum \hat{\sigma}_{a}^{2} \leq \delta^{2}$, and then consider the limit $\delta \rightarrow 0$ in a weak sense. The coefficients $c_{\delta}$ can always be chosen so that the limit exists and defines a Hilbert space isomorphic to the Dirac one [9]. Let the Hamiltonian have the form $\hat{H}=\hat{H}_{0}+\hat{V}$, where $V=V(x)$ is an everywhere regular potential and $\hat{H}_{0}=\hat{p}^{2} / 2$ is a kinetic energy operator. The operators $\hat{H}_{0}$ and $\hat{V}$ are assumed to be gauge invariant, i.e., they commute with the constraint operators ${ }^{3}$. Consider the evolution operator $\hat{U}_{T}=\exp (-i T \hat{H})$ in the total Hilbert space. The physical evolution operator is

\footnotetext{
${ }^{3}$ This assumption is justified for gauge theories of the Yang-Mills type. More general cases will be considered elsewhere because they are technically more involved and require a phase-space representation of quantum theory and a Wiener measure regularization of the corresponding path integral as proposed in [10.
} 
then $\hat{U}_{T}^{D}=\hat{U}_{T} \hat{\mathcal{P}}$. Making use of the Kato-Trotter product formula [11] for the evolution operator $\hat{U}_{T}$ we get the following product formula for the gauge invariant evolution operator

$$
\hat{U}_{T}^{D}=\left(e^{-i \epsilon \hat{H}_{0}} e^{-i \epsilon \hat{V}}\right)^{n} \hat{\mathcal{P}}=\left(e^{-i \epsilon \hat{H}_{0}} \hat{\mathcal{P}} e^{-i \epsilon \hat{V}}\right)^{n} \equiv\left(\hat{U}_{\epsilon}^{0 D} e^{-i \epsilon \hat{V}}\right)^{n} \equiv\left(\hat{U}_{\epsilon}^{D}\right)^{n},
$$

where $\epsilon=T / n$ and $n \rightarrow \infty$. In our previous work [10] we have shown that in the continuum limit the averaging gauge variables $\omega$ play the role of the Lagrange multipliers for the constraints in the classical action. By making use of the classical theory of Kolmogorov, we gave an explicit construction of a countably additive probability measure for the gauge functions $\omega(t)$. One natural choice was such that any set of values of $\omega(t)$ at any set of distinct times is equally likely. Now we show that this concept leads to a modification of the Faddeev-Popov procedure.

First we observe that by construction

$$
U_{T}^{D}\left(x, \Omega x^{\prime}\right)=U_{T}^{D}\left(\Omega x, x^{\prime}\right)=U_{T}^{D}\left(x, x^{\prime}\right)
$$

that is, the projected amplitude determines a genuine transition amplitude on the orbit space $\mathcal{M}$. To obtain its relation to the conventional gauge fixed amplitude, we introduce a set of local coordinates $h$ on $\mathcal{M}$ via a change of variables

$$
x=\Omega(\omega) h,
$$

where the configurations $x=h$ satisfy a gauge condition $\chi(x)=0$, i.e., $h \in \mathcal{H} \subset \mathcal{X}$ and without loss of generality we may assume $\mathcal{H} \sim \mathbb{R}^{M}$ (unless specified otherwise) with $M$ being the number of physical degrees of freedom. The gauge invariant amplitude (4) can be reduced on the gauge fixing surface $\mathcal{H}$ : $U_{T}^{D}\left(x, x^{\prime}\right)=U_{T}^{D}\left(h, h^{\prime}\right)$. This leads to a necessary

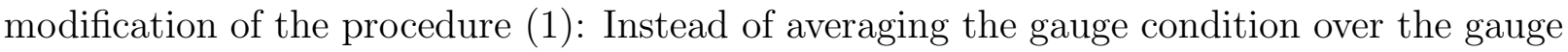
group, we propose to average an infinitesimal evolution operator kernel for a free motion as follows from the modified Kato-Trotter product formula (3)

$$
U_{\epsilon}^{0 D}\left(h, h^{\prime}\right)=(2 \pi i \epsilon)^{-N / 2} \int d \mu(\omega) e^{i\left(h-\Omega(\omega) h^{\prime}\right)^{2} / 2 \epsilon} .
$$

Since $\epsilon \rightarrow 0$, the averaging integral can be done to proper order in $\epsilon$ explicitly by the stationary phase approximation. The kernel $U_{\epsilon}^{D}\left(h, h^{\prime}\right)$ is obtained by adding $-i \epsilon V(h)$ to the exponential in (6) in accordance with (3) because $V(x)=V(h)$ thanks to the gauge invariance of $V$.

Suppose there exist transformations of the new variables $\omega$ and $h$ in (5), $\omega \rightarrow \omega+\omega_{s}$ and $h \rightarrow h_{s}$, such that $x(\omega, h)=x\left(\omega+\omega_{s}, h_{s}\right)$, where all configurations $x_{s}=h_{s}$ also satisfy the gauge condition $\chi(h)=\chi\left(h_{s}\right)=0$. From $\Omega\left(\omega+\omega_{s}\right) h_{s}=\Omega(\omega) h$ follows that $h_{s}=\Omega_{s} h$, i.e., $h_{s}$ is a Gribov copy of $x=h$ on the gauge fixing surface $\chi(x)=0$. If $\mathbb{S}$ is a collection of all residual gauge transformations, the modular domain $K$ is the quotient $\mathcal{H} / \mathbb{S}$. The relation (5) determines a one-to-one correspondence if $h \in K$ and $\omega$ parameterizes a group manifold. If $h, h^{\prime} \in K$, then the following relation holds

$$
U_{T}^{D}\left(h, h_{s}^{\prime}\right)=U_{T}^{D}\left(h_{s}, h^{\prime}\right)=U_{T}^{D}\left(h, h^{\prime}\right) .
$$


It determines an $\mathbb{S}$ - invariant continuation of the transition amplitude to the covering space $\mathcal{H}$ of the modular domain $K$. The Faddeev-Popov determinant specifies the volume element on $\mathcal{M}$ [5], so for any integral involving only functions on $\mathcal{M}, f(x)=f(\Omega x)$, one has $\int d x f(x)=\mathcal{V}_{G} \int_{K} d h \Delta_{F P}(h) f(h)$, where $\mathcal{V}_{G}$ is the volume of the gauge group. Therefore the folding of two projected infinitesimal evolution operators reads

$$
U_{2 \epsilon}^{D}\left(h, h^{\prime}\right)=\int_{K} d h_{1} \Delta_{F P}\left(h_{1}\right) U_{\epsilon}^{D}\left(h, h_{1}\right) U_{\epsilon}^{D}\left(h_{1}, h^{\prime}\right) .
$$

The restriction of the integration domain in (8) does not lead to a formal restriction of the integration domain in the path integral resulting from (3) in the continuum limit as one might naively expect from (8). An important point is that, if $K \neq \mathcal{H}$ (the Gribov problem is present), then $U_{\epsilon}^{0 D}\left(h, h^{\prime}\right)$ has no standard form of an infinitesimal free transition amplitude on a manifold proposed in [6] as we now proceed to demonstrate.

The stationary phase approximation can be applied before the reduction of $U_{\epsilon}^{D}\left(x, x^{\prime}\right)$ on a gauge fixing surface. A deviation from the conventional gauge-fixing procedure results from the fact that there may be more than just one stationary point. We can always shift the origin of the averaging variable $\omega$ so that one of the stationary points is at the origin $\omega=0$. Let $\hat{\boldsymbol{\delta}}_{a}$ be operators generating gauge transformations in $\mathcal{X}$. Decomposing the distance $(x-$ $\left.\Omega(\omega) x^{\prime}\right)^{2}$ in the vicinity of the stationary point, we find $\left(x-x^{\prime}, \hat{\boldsymbol{\delta}}_{a} x^{\prime}\right)=0$. So in the formal continuum limit we get the condition $\sigma_{a}(\dot{x}, x) \equiv\left(\dot{x}, \hat{\boldsymbol{\delta}}_{a} x\right)=0$ induced by the averaging procedure. This is nothing but the Gauss law enforcement for trajectories contributing to $U_{T}^{D}\left(x, x^{\prime}\right)$. Suppose there exists a gauge condition $\chi_{a}(x)=0$, which involves no time derivatives, such that a generic configuration $x=h$ satisfying it also fulfills identically the Gauss law, i.e., $\left(\dot{h}, \hat{\boldsymbol{\delta}}_{a} h\right) \equiv 0$. We will call it a natural gauge. In this case all other stationary points in the integral (6) are $\omega_{c}=\omega_{s}$ where $\Omega\left(\omega_{s}\right)=\Omega_{s} \in \mathbb{S}$. Therefore we get a sum over the stationary points in the averaging integral (6) if the Gribov problem is present. Still, in the continuum limit we have to control all terms of order $\epsilon$. This means that we need not only the leading term in the stationary phase approximation of (6) but also the next two corrections to it. Therefore the group element $\Omega(\omega)$ should be decomposed up to order $\omega^{4}$ because $\omega^{4} / \epsilon \sim \epsilon$ as one is easily convinced by rescaling the integration variable $\omega \rightarrow \sqrt{\epsilon} \omega$. The measure should also be decomposed up to the necessary order to control the $\epsilon$-terms. The latter would yield quantum corrections to the classical potential associated with the operator ordering in the kinetic energy operator on the orbit space. We stress that the averaging procedure gives a unique ordering so that the integral is invariant under general coordinate transformations on $\mathcal{M}$, i.e., does not depend on the choice of $\chi$ (cf. (14)). Thus,

$$
\begin{aligned}
U_{\epsilon}^{0 D}\left(h, h^{\prime}\right) & =(2 \pi i \epsilon)^{-M / 2} \sum_{\mathbb{S}} D^{-1 / 2}\left(h, h_{s}^{\prime}\right)\left[e^{i\left(h-h_{s}^{\prime}\right)^{2} / 2 \epsilon-i \epsilon \bar{V}_{q}\left(h, h_{s}^{\prime}\right)}+O\left(\epsilon^{2}\right)\right], \\
& \equiv \sum_{\mathbb{S}} D^{-1 / 2}\left(h, h_{s}^{\prime}\right) \tilde{U}_{\epsilon}\left(h, h_{s}^{\prime}\right),
\end{aligned}
$$

where $D\left(h, h^{\prime}\right)$ is the conventional determinant arising in the stationary phase approximation, and by $\bar{V}_{q}$ we denote a contribution of all relevant corrections to the leading order. The amplitude $U_{\epsilon}^{D}\left(h, h^{\prime}\right)$ is obtained by adding $-i \epsilon V(h)$ to the exponential in (9). Note that $V(h)=V\left(h_{s}\right)$ thanks to the gauge invariance of the potential. 
In general, the equations $\sigma_{a}(\dot{x}, x)=0$ are not integrable therefore the functions $\chi_{a}$ discussed above do not exist. In this case we consider two possibilities. Let $\Omega_{c}\left(h, h^{\prime}\right)$ be the group element at a stationary point in (6). Decomposing the distance in the vicinity of the stationary point we get $\left(h-\Omega_{c} h^{\prime}, \hat{\boldsymbol{\delta}}_{a} \Omega_{c} h^{\prime}\right)=0$. Let $\chi$ be such that the latter condition is also satisfied if $h^{\prime}$ is replaced by $h_{s}^{\prime}$. In this case the sum over the stationary points is again given by (9) where in the first term of the exponential $h_{s}^{\prime}$ is replaced by $\Omega_{c}\left(h, h_{s}^{\prime}\right) h_{s}^{\prime}$. In the most general case, the sum over stationary points does not coincide with the sum over Gribov copies. However for sufficiently small $\epsilon$, the averaged short-time transition amplitude can always be represented in the form (10). Note that as $\epsilon$ goes to zero, $U_{\epsilon}^{0}\left(x, x^{\prime}\right) \rightarrow \delta^{N}\left(x-x^{\prime}\right)$. If we change the variables in the delta function according to (5) and then do the gauge group average, we get, in general, a sum of delta functions on the gauge fixing surface. From the symmetry of the change of variables (5) it follows that the support of the averaged delta function is formed by points $h=h_{s}^{\prime}, h^{\prime} \in K$ and $h \in \mathcal{H}$, which means that the sum over $\mathbb{S}$ emerges again for sufficiently small $\epsilon$. We will explain below how to calculate relevant corrections to the leading order of the limit $\epsilon \rightarrow 0$.

Let us replace $U_{\epsilon}^{D}\left(h, h_{1}\right)$ in (8) by the sum (10) and make use of (7) applied to the second kernel in (8): $U_{\epsilon}^{D}\left(h_{1}, h^{\prime}\right)=U_{\epsilon}^{D}\left(h_{1 s}, h^{\prime}\right)$. Since the measure on the orbit space does not depend on a particular choice of the modular domain, $d h_{s} \Delta_{F P}\left(h_{s}\right)=d h \Delta_{F P}(h)$, we can extend the integration to the entire covering space by removing the sum over $\mathbb{S}$

$$
U_{2 \epsilon}^{D}\left(h, h^{\prime}\right)=\int d h_{1}\left|\Delta_{F P}\left(h_{1}\right)\right| D^{-1 / 2}\left(h, h_{1}\right) \tilde{U}_{\epsilon}\left(h, h_{1}\right) U_{\epsilon}^{D}\left(h_{1}, h^{\prime}\right) .
$$

The absolute value bars accounts for a possible sign change of the Faddeev-Popov determinant on the gauge fixing surface. The procedure can be repeated from left to right in the folding (3), thus removing the restriction of the integration domain and the sum over copies in all intermediate times $t \in(0, T)$. The sum over $\mathbb{S}$ for the initial configuration $h^{\prime}$ remains in the integral.

Now we can formally take a continuum limit with the result

$$
U_{T}^{D}\left(h, h^{\prime}\right)=\sum_{\mathbb{S}}\left[\Delta_{F P}(h) \Delta_{F P}\left(h_{s}^{\prime}\right)\right]^{-1 / 2} \int_{h(0)=h_{s}^{\prime}}^{h(T)=h} \mathcal{D} h \sqrt{\operatorname{det} g_{p h}} e^{i \int_{0}^{T} d t\left[\left(\dot{h}, g_{p h} \dot{h}\right) / 2-V_{q}(h)-V(h)\right]}
$$

where $g_{p h}$ is the induced metric on $\mathcal{M}$. The local density $\prod_{t} \sqrt{\operatorname{det} g_{p h}}$ should be understood as the result of the integration over the momenta in the corresponding time-sliced phasespace path integral where the kinetic energy is $\left(p_{h}, g_{p h}^{-1} p_{h}\right) / 2$ with $p_{h}$ being a canonical momentum for $h$. To obtain (12), one usually sets $h^{\prime}=h-\Delta$ for $h=h(t)$ and $h^{\prime}=$ $h(t-\epsilon)$ in each intermediate moment of time $t$ and makes a decomposition into the power series over $\Delta$. According to the relation, obtained in [5], between the volume of a gauge orbit through $x=h$, the induced metric $g_{p h}$, and the Faddeev-Popov determinant we get $D\left(h^{\prime}+\Delta, h^{\prime}\right)=\Delta_{F P}^{2}\left(h^{\prime}\right) / \operatorname{det} g_{p h}\left(h^{\prime}\right)+O(\Delta)$. This relation explains the cancellation of the absolute value of the Faddeev-Popov determinant in the folding (3) made in accordance with the rule (11). The term $\Delta^{2} / \epsilon$ in the exponential (9) gives rise to the kinetic energy $\left(\Delta, g_{p h} \Delta\right) / 2 \epsilon+O\left(\Delta^{3}\right)$. 
The most difficult part to calculate are the operator ordering corrections $V_{q}(h)$ in the continuum limit. Here we remark that $D\left(h, h^{\prime}\right)$ has to be decomposed up to order $\Delta^{2}$, while the exponential in (9) up to order $\Delta^{4}$ because the measure has support on paths for which $\Delta^{2} \sim \epsilon$ (i.e., $\Delta^{4} / \epsilon \sim \epsilon$ ). There is a technique, called the equivalence rules for Lagrangian path integrals on manifolds, which allows one to convert terms $\Delta^{2 n}$ into terms $\epsilon^{n}$ and thereby to calculate $V_{q}$ [6, 12]

$$
\Delta^{j_{1}} \cdots \Delta^{j_{2 k}} \rightarrow(i \epsilon \hbar)^{k} \sum_{p\left(j_{1}, \ldots, j_{2 k}\right)}\left(g_{p h}^{-1}\right)^{j_{1} j_{2}} \cdots\left(g_{p h}^{-1}\right)^{j_{2 k-1} j_{2 k}},
$$

where the sum is extended over all permutations of the indices $j$ to make the right-hand side of (13) symmetric under permutations of the $j$ 's. Following (13) one derives the Schrödinger equation for the physical amlitude (12). The corresponding Hamiltonian operator on the orbit space has the form

$$
\hat{H}_{p h}=-1 / 2 \Delta_{F P}^{-1} \partial_{j}\left(\left[g_{p h}^{-1}\right]^{j k} \Delta_{F P} \partial_{k}\right)+V(h),
$$

where $\partial_{j}=\partial / \partial h_{j}$. Observe that the kinetic energy in (14) does not coincide with the Laplace-Beltrami operator on $\mathcal{M}$ because $\left[\operatorname{det} g_{p h}\right]^{1 / 2} \neq \Delta_{F P}$ as shown in [5]. The operator (14) is hermitian with respect to the scalar product where the volume element is $d h \Delta_{F P}$. It is also invariant under general coordinate transformations on $\mathcal{M}$, i.e., its spectrum does not depend on the choice of local coordinates on $\mathcal{M}$ and, therefore, is gauge invariant. We skip technical details of the derivation of (14) because they are standard in the path integral formalism and instead turn to examples to illustrate the main features of the new projection formalism. But before we do so, let us comment that the effects on the energy spectrum caused by the modification of the path integral can be found from the pole structure of the trace of the resolvent

$$
\begin{aligned}
\operatorname{tr} \hat{R}(\tau) & =\operatorname{tr}(\tau-i \hat{H})^{-1}=\int_{0}^{\infty} d T e^{-\tau T} \operatorname{tr} \hat{U}_{T}^{D}, \\
\operatorname{tr} \hat{U}_{T}^{D} & =\int_{K} d h \Delta_{F P}(h) U_{T}^{D}(h, h) .
\end{aligned}
$$

3. Examples. Let us take the simplest example [4] where $x \in \mathbb{R}^{3}$ and the gauge transformations are rotations of $x$ about the origin. The orbit space is a space of concentric spheres. The natural gauge is $x^{i}=\delta^{i 1} h$. This gauge is not complete because there are rotations that change the sign of $h: h \rightarrow \pm h$. These transformations form the group $\mathbb{S}=\mathbb{Z}_{2}$. The modular domain is an open half-axis $K=(0, \infty)$. The measure on the orbit space is obtained by introducing spherical coordinates on $\mathbb{R}^{3}$ so that $h=|x|$, while the spherical angles parameterize the gauge group manifold $S O(3) / S O(2)$. It is noteworthy to observe that in the projection formalism there is no principal difference between reducible and irreducible gauge theories. Here we obviously have a reducible case because $x$ has a stationary group $S O(2)$. The Faddeev-Popov determinant is defined with respect to a subset of independent constraints. In this case, the constraints are three components of the angular momentum. So we can take the angular momenta about the axes $i=2,3$ as independent constraints and find that $\Delta_{F P}=h^{2}$ in the full accordance with the measure resulting from the spherical 
coordinates. The angular measure must be normalized by $4 \pi$ to fulfill the condition in (2). So Eq. (6) assumes the form

$$
U_{\epsilon}^{0 D}\left(h, h^{\prime}\right)=(2 \pi i \epsilon)^{-3 / 2} 1 / 2 \int_{0}^{\pi} d \omega \sin \omega e^{i\left(h^{2}+h^{\prime 2}\right) / 2 \epsilon-i h h^{\prime} \cos \omega / \epsilon} .
$$

Doing the averaging integral exactly and substituting the result into (3) we obtain

$$
U_{T}^{D}\left(h, h^{\prime}\right)=\left[h h^{\prime}\right]^{-1}\left\{U_{T}\left(h, h^{\prime}\right)-U_{T}\left(h,-h^{\prime}\right)\right\},
$$

where the amplitude $U_{T}\left(h, h^{\prime}\right)$ is given by a standard path integral for a one-dimensional system with the gauge-fixed action $S_{g f}[h]=\int_{0}^{T} d t\left[\dot{h}^{2} / 2-V(h)\right]$. There is no operator ordering correction.

The stationary phase approximation leads to the same result if one takes into account all the stationary points, i.e., $\omega_{s}=0$ and $\omega_{s}=\pi$. The second stationary point is associated with the only nontrivial residual gauge transformation $h^{\prime} \rightarrow-h^{\prime}$. Here one also gets $\bar{V}_{q}=0$ (cf. (9)). The same path integral can be derived from the Schrödinger equation projected on the orbit space [13]. It is not hard to calculate the partition function (16) for a harmonic oscillator $V=x^{2} / 2=h^{2} / 2$ and find the resolvent (15). The poles determine the spectrum $E_{n}=2 n+3 / 2$. The distance between the energy levels is 2 , while the oscillator frequency in the Hamiltonian is 1 . The effect of the doubling would be lost, had we ignored the redundant discrete gauge symmetry and calculated the partition function by a standard path integral for the gauge-fixed action $S_{g f}$.

A simple model to illustrate the effect of the residual gauge symmetry in systems with several physical degrees of freedom is a Yang-Mills theory in $(1+0)$ spacetime [13]. A total configuration space is a Lie algebra $X$, and the gauge group acts in the adjoint representation $x \rightarrow \Omega x \Omega^{-1}$. The quadratic form in the exponential (6) is taken with respect to the Killing form on $X:(x, x) \equiv \operatorname{tr} \hat{x}^{2}$, where $\hat{x} y \equiv[x, y]$ for any $x, y \in X$ and the commutator stands for the Lie bracket in $X$. If a matrix representation is assumed, for compact groups it can always be normalized to the ordinary matrix trace. The Gauss law is $\sigma=[\dot{x}, x]=0$, therefore the natural gauge condition to parameterize $\mathcal{M}$ is $x=h \in H$ where $H$ is a Cartan subalgebra (a maximal commutative subalgebra of $X$ ). The system has $r=\operatorname{dim} H$ physical degrees of freedom. The invariant measure $d \mu(\omega)$ is proportional to $d \omega \operatorname{det}\left[(\exp \hat{\omega}-1) \hat{\omega}^{-1}\right]$. In the exponential in $(6)$ we get $\left(h-\exp (\hat{\omega}) h^{\prime}\right)^{2}$. An adjoint transformation of $x$ to $H$ is not unique and determined modulo the Weyl group $\mathbb{W}$ acting in $H$. The Weyl transformations are inequivalent compositions of reflections in the hyperplanes orthogonal to simple roots in $H \llbracket 19]$. So $\mathbb{S}=\mathbb{W}$ and $\mathcal{M} \sim H / \mathbb{W}=K^{+}$, where $K^{+}$is the Weyl chamber in $H$. The stationary points of the integral (6) are $\omega_{c}=\omega_{W}$ where the adjoint action of the group element $\exp \omega_{W}$ on $h$ transforms the latter by a Weyl reflection. Introducing the Cartan-Weyl basis [19] one can find the Jacobian of the change of variables (5) (the Faddeev-Popov determinant) $\Delta_{F P}(h)=\operatorname{det} \hat{h}=\kappa^{2}(h)$ where $\kappa(h)=\prod_{\alpha>0}(\alpha, h)$; the product is extended over all positive roots in $H$. The operator $\hat{h}$ acts in the orthogonal complement of the Cartan subalgebra. Note that the Cartan group is the stationary group of $h \in H$, so $\omega$ should take its values in the orthogonal supplement of the Cartan subalgebra. The factor $D\left(h, h^{\prime}\right)$ is given by the determinant of the operator $\hat{h} \hat{h}^{\prime}$. Doing the stationary 
phase approximation in the averaging integral, and then taking the folding (3) we find

$$
U_{T}^{D}\left(h, h^{\prime}\right)=\sum_{\mathbb{W}}\left[\kappa(h) \kappa\left(h_{W}^{\prime}\right)\right]^{-1} U_{T}\left(h, h_{W}^{\prime}\right) .
$$

Here the amplitude $U_{T}\left(h, h^{\prime}\right)$ is given by the conventional path integral (no restriction of the integration domain to the Weyl chamber) with the action $\int d t\left(\dot{h}^{2} / 2-V(h)\right)$. There is no operator ordering correction, and the physical metric is Euclidean. The same integral follows from a direct solution of the Schrödinger equation [13]. The Faddeev-Popov determinant vanishes at the hyperplanes $(h, \alpha)=0$. The boundary of the modular domain (the Weyl chamber) is formed by these hyperplanes when $\alpha$ runs over the simple roots. The amplitude (19) remains finite at the singular points (the Gribov horizon) because $\kappa\left(h_{W}\right)=p_{W} \kappa(h)$ where $p_{W}= \pm 1$ is the parity of the corresponding Weyl transformation (cf. also (18): It remains finite at $h=0$ or $\left.h^{\prime}=0\right)$ ). Contributions of paths hitting the horizon have a dramatic effect on the spectrum of the isotropic oscillator $V=x^{2} / 2=h^{2} / 2$. Doing the path integral for $U_{T}\left(h, h^{\prime}\right)$ in (19) and calculating the poles of the resolvent (15) we find 113 $E_{n}=\nu_{1} n_{1}+\cdots+\nu_{r} n_{r}+N / 2$, where $n_{i}$ are non-negative integers, $\nu_{i}$ are orders of invariant irreducible symmetric tensors of the Lie algebra, and $r$ and $N$ are, respectively, the rank and dimension of the group (e.g., for $\left.\mathrm{SU}(r+1), \nu_{i}=i+1, i=1,2, \ldots, r\right)$. Thus, the physical frequencies of the isotropic oscillator appear to be $\nu_{i} \neq 1$. The partition function calculated by a standard path integral for the gauge fixed action of the model would lead to the spectrum of the isotropic oscillator (i.e., $\nu_{i} \rightarrow 1$ ).

To illustrate the effects of curvature of the orbit space, we consider a simple gauge matrix model. Let $x$ be a real $2 \times 2$ matrix subject to the gauge transformations $x \rightarrow \Omega(\omega) x$ where $\Omega \in S O(2)$. An invariant scalar product reads $\left(x, x^{\prime}\right)=\operatorname{tr} x^{T} x^{\prime}$ with $x^{T}$ being a transposed matrix $x$. The total configuration space is $\mathbb{R}^{4}$. Let $\varepsilon$ be a generator of $\operatorname{SO}(2)$, i.e., $\varepsilon_{i j}=-\varepsilon_{j i}$, and $\varepsilon_{12}=1$. Then $\Omega(\omega)=\exp (\omega \varepsilon)$. The Gauss law enforced by the projection is $\sigma=\operatorname{tr} \dot{x}^{T} \varepsilon x=0$. It is not integrable. We parameterize $\mathcal{M}$ by triangular matrices $h_{21} \equiv 0$ (the gauge $x_{21}=0$ ). The residual gauge transformations form the group $\mathbb{S}=\mathbb{Z}_{2}: h \rightarrow \pm h$. The modular domain is a positive half-space $h_{11}>0$. Calculating the Jacobian of the change of variables (5) for this model we find the Faddeev-Popov determinant $\Delta_{F P}(h)=h_{11}$. The plane $h_{11}=0$ is the Gribov horizon. The averaging measure in (6) reads $(2 \pi)^{-1} d \omega$, where $\omega \in[0,2 \pi)$. The quadratic form in the exponential in (6) is

$$
\left(h-e^{\omega \varepsilon} h^{\prime}\right)^{2}=(h, h)+\left(h^{\prime}, h^{\prime}\right)-2\left(h, h^{\prime}\right) \cos \omega-2\left(h, \varepsilon h^{\prime}\right) \sin \omega .
$$

A distinguished feature of this model from those considered above is that the stationary point is a function of $h$ and $h^{\prime}$. Taking the derivative of (20) with respect to $\omega$ and setting it to zero, we find

$$
\omega_{c}=\tan ^{-1} \frac{\left(h, \varepsilon h^{\prime}\right)}{\left(h, h^{\prime}\right)}, \quad \omega_{c}^{s}=\omega_{c}+\pi .
$$

The second stationary point $\omega_{c}^{s}$ is associated with the Gribov transformation $h \rightarrow-h$. A geometrical meaning of the transformation $h^{\prime} \rightarrow \exp \left(\omega_{c} \varepsilon\right) h^{\prime}$ is transparent. The distance $\left[\left(h-h^{\prime}\right)^{2}\right]^{1 / 2}$ between two points on the gauge fixing plane is greater than the minimal distance between the two gauge orbits through $x=h$ and $x^{\prime}=h^{\prime}$. By shifting $x^{\prime}$ along the 
gauge orbit to $x_{c}^{\prime}=\exp \left(\omega_{c} \varepsilon\right) h^{\prime}$ a minimum of the distance between the orbits is achieved. In such a way the metric on the orbit space emerges in the projection formalism. Its explicit form can be found as has been explained in section 2. We substitute $\omega=\omega_{c}\left(h, h^{\prime}\right)$ into (20), set $h^{\prime}=h-\Delta$ and decompose (20) in a power series over $\Delta$. The quadratic term (the leading term) determines the metric. We get $\left(\Delta, g_{p h}(h) \Delta\right)=(\Delta, \Delta)+(\Delta, \varepsilon h)(\varepsilon h, \Delta) /(h, h)$. The metric is not flat. The scalar Riemann curvature of $\mathcal{M}$ is $R=6 /(h, h)=6 /(x, x)$. Note that it is gauge invariant as it should be because the curvature $R$ does not depend on the choice of local coordinates on $\mathcal{M}$. In the stationary phase approximation the cosine and sine in (20) should be decomposed up to fourth order in the vicinity of the stationary point. In this model quantum corrections do not vanish. The infinitesimal transition amplitude on $\mathcal{M}$ assumes the form

$$
\begin{aligned}
U_{\epsilon}^{D}\left(h, h^{\prime}\right) & =(2 \pi i \epsilon)^{-3 / 2}\left[D^{-1 / 2}\left(h, h^{\prime}\right) e^{i S_{\epsilon}\left(h, h^{\prime}\right)}+D^{-1 / 2}\left(h,-h^{\prime}\right) e^{i S_{\epsilon}\left(h,-h^{\prime}\right)}\right], \\
S_{\epsilon}\left(h, h^{\prime}\right) & =\left[(h, h)+\left(h^{\prime}, h^{\prime}\right)-2 D\left(h, h^{\prime}\right)\right] /(2 \epsilon)-\epsilon\left[8 D\left(h, h^{\prime}\right)\right]^{-1}-\epsilon V(h),
\end{aligned}
$$

where $D\left(h, h^{\prime}\right)=\left(h, h^{\prime}\right) \cos \omega_{c}+\left(h, \varepsilon h^{\prime}\right) \sin \omega_{c}$. Observe that the middle term in (23) is a quantum correction to the classical potential in the short-time action (23) (it is proportional to $\hbar^{2}$, if one restores the Planck constant). The two first terms in (23) determine (uniquely!) the operator ordering in the kinetic energy operator on the orbit space in accordance with (14).

Finally, we consider the case of an infinite number of stationary points in the averaging integral (6). This would have an effect that the modular domain $K$ may become compact, which, in turn, leads to a discrete spectrum regardless of the details of the potential and therefore gives a mechanism for the gap between the ground state and the first excited state. A suitable soluble model is the Yang-Mills theory in cylindrical space time [14, 15, 16] where space is compactified into a circle. A Yang-Mills theory without matter in two dimensions has no physical degrees of freedom unless the spacetime has a nontrivial topology. The latter is exactly our case. The total configuration space $\mathcal{X}$ is the space of all periodic connections $A(x+2 \pi)=A(x)$ taking their values in the Lie algebra of a compact Lie group. The gauge group $\mathcal{G}$ acts on $\mathcal{X}$ as $A \rightarrow{ }^{\Omega} A=\Omega A \Omega^{-1}-i \Omega \partial \Omega^{-1}$, and $\Omega(x+2 \pi)=\Omega(x)$ (i.e., the gauge group elements $\Omega$ can be continuously deformed towards the group unit [17, 18]). The exponential in (6) assumes the form $\int_{0}^{2 \pi} d x \operatorname{tr}\left(A-{ }^{\Omega} A^{\prime}\right)^{2}$ for any two configurations $A(x)$ and $A^{\prime}(x)$. It is the distance between $A(x)$ and ${ }^{\Omega} A^{\prime}(x)$ introduced by Feynman [8]. A rigorous definition of the averaging integral over $\mathcal{G}$ can be given within the Kogut-Susskind lattice gauge theory, to which we turn shortly. Note, for example, that $N$ in (6) is infinite because the number of degrees of freedom is determined by the number of Fourier modes of $A(x)$.

Here we assume the existence of a normalized averaging measure on $\mathcal{G}$. The gauge group average enforces the Gauss law $\sigma=\partial \dot{A}-i[A, \dot{A}] \equiv \nabla(A) \dot{A}=0$. The orbit space $\mathcal{M}$ can be parameterized by constant connections $A(x)=h$ taking their values in the Cartan subalgebra $H$ [14, 16]. This is the natural gauge because $\sigma(h, h) \equiv 0$. The number of the physical degrees of freedom equals the rank of the gauge group. We set $\Omega(x)=\exp (i \omega(x))$. The averaging function $\omega$ takes its values in the Lie algebra and its constant (independent of $x$ ) Fourier mode does not have any component in the Cartan subalgebra because the connection $A(x)=h$ is invariant under constant gauge transformations from the Cartan 
subgroup. The Gaussian integral over such functions can formally be done in (6) yielding $D^{-1 / 2}\left(h, h^{\prime}\right)=\operatorname{det}^{-1 / 2}\left[-\nabla(h) \nabla\left(h^{\prime}\right)\right]$ which is easy to compute in the Cartan-Weyl basis [16. In the lattice formulation described below this procedure would not involve any infinite factors in $D\left(h, h^{\prime}\right)$ as in the formal continuum limit. Here we draw attention to the following feature. A projection of any connection $A(x)$ into the subspace of constant Cartan connections $h$ is not unique and determined modulo the affine Weyl transformations [16]. The affine Weyl group $\mathbb{W}_{A}$ is a semidirect product of the Weyl group $\mathbb{W}$ and the group of translation along the group unit lattice in the Cartan subalgebra [19]. Thus, the number of stationary points in the averaging integral (6) is infinite because the number of elements $\mathbb{W}_{A}$ is infinite: $h \rightarrow h_{s} \equiv h_{W}+2 \alpha n_{\alpha} /(\alpha, \alpha)$, where $h_{W}$ is a Weyl transformation of $h, \alpha$ any positive root, $n_{\alpha}$ an integer. The modular domain $K=H / \mathbb{W}_{A}$ is called the Weyl cell 119. It is compact in $H$. This has a significant effect on the spectrum of the system. In the continuum limit the transition amplitude has the form (19) where the sum is extended over the affine Weyl group (over an infinite number of stationary points $h_{s}^{\prime}$ ) [16], the FaddeevPopov determinant reads $\Delta_{F P}(h)=\kappa^{2}(h)$ and $\kappa(h)=\prod_{\alpha>0} \sin (h, \alpha)$. There is no potential energy in $1+1$ dimensional Yang-Mills theory $(V(h) \equiv 0$ in (19)). Thus, we get an amplitude for a free particle symmetrized over the affine Weyl group.

In the simplest $\mathrm{SU}(2)$ case, the system has only one physical degree of freedom. The Weyl transformations are reflections $h_{W}= \pm h$ and the translations over the group unit lattice are shifts $h \rightarrow h+2 n$. The amplitude (19) appears similar to the one for a particle in an infinite well or on a circle. The spectrum of such a system is discrete. In general, we can calculate the poles of the resolvent (15) for the 2D Yang-Mills theory using our path integral formalism and find [16] the spectrum $E_{n}=E_{0}\left[\left(\gamma_{n}, \gamma_{n}\right)-(\rho, \rho)\right]$ where $E_{0}$ is a constant, $\rho$ is a half the sum of all positive roots, and $\gamma_{n}$ ranges over a lattice in the Weyl chamber that labels all irreducible representation of the gauge group.

Thus, an infinite number of stationary points in the averaging integral (6) is, in fact, evidence that the physical configuration space (or its subspace) is compact. This is the mathematical structure of the path integral which is to be sought when studying the relation between the mass gap in gluodynamics and the compactness of the orbit space. Physically, the existence of an infinite number of the stationary points separated by different distances (large and small) implies that physical eigenstates of the Hamiltonian should exhibit a periodicity in some directions in $\mathcal{X}$, which may cause the mass gap as has been argued by Feynman [8].

4. Kogut-Susskind lattice gauge theory. To fulfill our program in realistic YangMills theory, we need a regularization at short distances to give a rigorous meaning to the averaging integral (6) which is a functional integral. The simplest possibility is to set the system on a lattice. Since we still want to have a Hamiltonian formalism, the time should remain continuous. With such a choice of the regularization we arrive at the Kogut-Susskind lattice gauge theory [20].

Let $y$ be a three-vector whose components are integer-valued in the lattice spacing unit $a$, i.e., $y$ labels the lattice sites. With each link connecting the lattice sites $y$ and $y+i a$, where $i$ is the unit vector in the direction of the $i$ th coordinate axis, we associate a group element $u_{y, i}$ called the link variable. The collection of all values of all link variables 
forms the configuration space $\mathcal{X}$ of the system. The gauge group $\mathcal{G}$ acts on $\mathcal{X}$ by the rule $u_{y, i} \rightarrow \Omega_{y} u_{y, i} \Omega_{y+i a}^{-1}$. Therefore the averaging measure is a product of the normalized Haar measures at each site: $d \mu=\prod_{y} d \mu_{H}\left(\Omega_{y}\right)$. The Kogut-Susskind Hamiltonian is $H=H_{0}+V$ where the potential $V$ is nothing but the color magnetic energy on the lattice. The kinetic energy is the sum of kinetic energies of a rigid rotators associated with each link variable

$$
H_{0}=1 / 2 \sum_{y, i} \operatorname{tr}\left(\dot{u}_{y, i} u_{y, i}^{-1}\right)^{2} .
$$

An important observation is that the coupling of the rotators occurs only through the potential $V$. Therefore the "free" amplitude $U_{T}^{0}\left(\{u\},\left\{u^{\prime}\right\}\right)$, where $\{u\}$ is a collection of configurations of rotators $u_{y, i}$, is factorized into a product of the amplitudes for each rotator $U_{T}^{0}\left(u_{y, i}, u_{y, i}^{\prime}\right)$. An explicit form of the latter is well-known [21] (a transition amplitude of a particle moving on a group manifold). This replaces the free motion amplitude in a Euclidean space in the averaging integral (6). The averaging integral

$$
U_{\epsilon}^{0 D}\left(\{u\},\left\{u^{\prime}\right\}\right)=\int \prod_{y}\left[d \mu_{H}\left(\Omega_{y}\right)\right] \prod_{y, i} U_{\epsilon}^{0}\left(u_{y, i}, \Omega_{y} u_{y, i}^{\prime} \Omega_{y+a i}\right)
$$

can be calculated via both the stationary phase approximation and the decomposition over the characters of the irreducible representations of the amplitudes $U_{T}^{0}\left(u, u^{\prime}\right)$ proposed in 21. Thus, we have reduced the problem of constructing the transition amplitude on the orbit space to a standard mathematical problem in group theory. It is important that the effects of the Riemann structure (metric) and topology of the orbit space are completely determined by the averaging procedure proposed and do not depend on the details of the self-interaction $V$. Note that the average in (25) induces a nontrivial coupling between the rotators because six link variables attached to the same site are transformed with the same group element. So the factorization of the amplitude on $\mathcal{M}$ is no longer possible. This is the result of the enforcement of the Gauss law in the physical amplitude.

Thus, we get, in principle, a constructive and consistent procedure to study the effects of the non-Euclidean geometry of the orbit space on physical quantities like the spectrum of low energy excitations. In particular, in the strong coupling limit ( $g$ large) the potential $V$ can be treated by perturbation theory [20]. To introduce an explicit parameterization of the orbit space, one can, for example, make use of Morse theory for the Kogut-Susskind lattice gauge theory as proposed in [22]. In this case, the initial configuration $\left\{u^{\prime}\right\}$ in the averaging integral (25) is taken from the corresponding modular domain. The projected amplitude has a natural gauge invariant continuation to the covering space of the modular domain.

5. Conclusions. We have proposed a self-consistent path integral quantization of gauge theories which resolves the Gribov obstruction and provides control for the operator ordering problem, and it does not rely explicitly on the Schrödinger equation on the orbit space. This was our main goal. The continuum limit in the Kogut-Susskind lattice gauge theory might still be a difficult analytical problem. However, it is believed that the techniques we have developed might be useful to study the effects of the non-Euclidean geometry and topology of the orbit space in some physically reasonable approximations. We have also demonstrated its effectiveness with several examples. 


\section{References}

[1] L.D. Faddeev and V.N. Popov, Phys. Lett. B25 (1968) 30.

[2] V.N. Gribov, Nucl. Phys. B139 (1978) 1.

[3] I. Singer, Commun. Math. Phys. 60 (1978) 7.

[4] N. Christ and T.D. Lee, Phys. Rev. D22 (1980) 939.

[5] O. Babelon and Viallet, Phys. Lett. B85 (1979) 246.

[6] B. De Witt, Rev. Mod. Phys. 29 (1957) 377.

[7] J. Zinn-Justin, Quantum Field Theory and Critical Phenomena, 2nd Edition, Clarendon Press, Oxford, 1990.

[8] R. Feynman, Nucl. Phys. B188 (1981) 479.

[9] J.R. Klauder, Ann. Phys. (NY) 254 (1997) 419.

[10] J.R. Klauder and S.V. Shabanov, Phys. Lett. B 398 (1997) 116.

[11] E. Nelson, J. Math. Phys. 5 (1964) 332;

T. Kato and H.F. Trotter, Pacific Math. J. 8 (1959) 545.

[12] L.V. Prokhorov, Sov. J. Part. Nucl. 13 (1982) 456.

[13] L.V. Prokhorov and S.V. Shabanov, Phys. Lett. B216 (1989) 292;

Sov. Phys. Uspekhi 34 (1991) 108.

[14] S.G. Rajeev, Phys. Lett. B212 (1989) 203.

[15] E. Langman and G.W. Semenoff, Phys. Lett. B303 (1993) 303.

[16] S.V. Shabanov, Phys. Lett. B318 (1993) 323.

[17] G. 't Hooft, Nucl. Phys. B153 (1979) 141.

[18] R. Jackiw, Rev. Mod. Phys. 52 (1980) 661.

[19] S. Helgason, Differential Geometry, Lie Groups, and Symmetric Spaces, Academic Press, New York, 1978.

[20] J. Kogut and L. Susskind, Phys. Rev. D11 (1976) 395.

[21] M.S. Marinov and M.V. Terentiev, Fortshcr. Phys. 27 (1979) 511.

[22] D. Zwanziger, Nucl.Phys. B485 (1997) 185. 